\title{
Geschäftsführung und Aufsicht trennen
}

\section{Die Grundsätze verantwortungsvoller Unternehmensführung im Deutschen Caritasverband}

Christopher Bangert

Dr. Christopher Bangert ist Leiter der Stabsstelle Sozialwirtschaft des Deutschen Caritasverbandes in Freiburg im Breisgau.

E-Mail

christopher.bangert@caritas.de
Wenn soziale Dienste und Einrichtungen sorgsam mit ökonomischen Ressourcen umgehen sollen, müssen sich die Verantwortlichen im wirtschaftlichen Bereich wie ordentliche Kaufleute verhalten. Dieser Ansatz liegt den Regeln zugrunde, die sich der Deutsche Caritasverband gegeben hat. Ziel ist dabei, durch ein höheres Maß an Transparenz die Kontroll- und Überwachungsfunktion im Unternehmen zu stärken.

Spätestens seit der Veröffentlichung des ersten Deutschen Corporate Governance Kodex (DCGK) im Februar 2002 sehen sich deutsche Unternehmen mit einem höheren Rechtfertigungs- und Transparenzdruck konfrontiert. Dabei beschränkt sich die Wirkung des Kodex inzwischen nicht mehr alleine auf die eigentliche Zielgruppe, die börsennotierten Unternehmen. Dahinter verbirgt sich die Einsicht, dass alle Unternehmen in der Öffentlichkeit stehen - auch die gemeinnützigen Sozialunternehmen - und sich einem Transparenzinteresse von externen Adressaten wie Klienten, Patienten, Angehörige, Spendern, Kapitalgebern, Kostenträgern und Aufsichtsbehörden gegenübersehen. Deshalb sollten sie die Grundsätze der guten und verantwortungsvollen Unternehmensführung ebenso anwenden wie die gewerblichen Unternehmen.

Es gibt gute Gründe dafür, warum sich die verbandliche Caritas dieser gesellschaftlichen Anforderungen stellen und Maßnahmen im eigenen Bereich ergreifen sollte. Mit ihren gut 24.000 Einrichtungen und Diensten stellt die verbandliche Caritas ein wesentliches Rückgrat für die soziale Infrastruktur in Deutschland dar. Sie ist ein bedeutender Arbeitgeber: Die circa 10.000 selbstständigen Rechtsträger unter dem Dach der Caritas beschäftigen rund 507.000 hauptamtliche Mitarbeiterinnen und Mitarbeiter. Diese Zahlen verdeutlichen, dass die verbandliche Caritas auch ein bedeutsamer Wirtschaftsfaktor ist.
Einige größere und medienwirksame wirtschaftliche Zusammenbrüche von katholischen Trägern aus der Sozialwirtschaft, die vor Jahren aufgetreten sind, haben gezeigt, dass die Caritas ihrer Verantwortung besser gerecht werden muss. Der Umgang mit dem Wettbewerb und den sich verschärfenden Refinanzierungsbedingungen ist zum Alltag geworden. Inzwischen haben wir es im Bereich der Caritas mit Fakten und Bedingungen zu tun, die mit denen der gewerblichen Wirtschaft vergleichbar sind.

Zum finanziellen Schaden von Unternehmenskrisen kommt ein nicht unerheblicher Imageschaden, der Kirche und Caritas durch diese Bankrotte trifft. In dieser Situation stehen die Kirche und ihre Caritas vor der grundsätzlichen Frage, wie man zumindest die Wahrscheinlichkeit, dass weitere wirtschaftliche Pleiten kirchlicher und karitativer Träger eintreten, minimieren kann. Die Etablierung einer qualifizierten Aufsicht und Kontrolle tritt dabei in den Vordergrund.

Dabei gilt es, die bewährte dezentrale gemeinde- und wohnortnahe Struktur kirchlich-karitativer Einrichtungen und Dienste nach dem Subsidiaritätsprinzip zu bewahren. Falsch wäre es nach mehr Zentralität und nach (noch) mehr staatlicher und kirchlicher Aufsicht zu rufen. Vielmehr ist es notwendig, die Verantwortung der Rechtsträger in den Mittelpunkt zu stellen, und den Trägern verbandliche Unterstützung bei der Implementierung von effektiven Aufsichtsstrukturen anzubieten.

\section{Handlungsgrundlage}

Die katholische Kirche und ihre Caritas konnten sich bei ihren Bemühungen um die Einführung einer effektiven Aufsicht und Kontrolle an Bewährtem orientieren. Wenn die Verantwortlichen der Rechtsträger der Caritas im wirtschaftlichen Bereich wie ordentliche Kaufleute handeln 
sollen, liegt die Übernahme und Adaption von bewährten Regelen aus dem gewerblichen Bereich nahe. Dieser Ansatz liegt der »Arbeitshilfe 182 « des Verbandes der Diözesen Deutschlands und der Kommission für caritative Fragen der Deutschen Bischofskonferenz mit dem Titel »Soziale Einrichtungen in katholischer Trägerschaft und wirtschaftliche Aufsicht « zugrunde, die erstmals 2004 und in zweiter Auflage Anfang 2007 erschienen ist. Gemäß der Arbeitshilfe sollen die Rechtsträger der Caritas die Grundsätze des Deutschen Corporate Governance Kodex, die teilweise auch schon ins Aktien- und GmbH-Gesetz eingeflossen sind, im Sinne einer Selbstverpflichtung übernehmen. (1) Diese Selbstverpflichtung gilt insbesondere für Träger mit mehr als 50 Mitarbeitenden, aber auch kleinere Träger sollten die Umsetzung ernsthaft prüfen.

Dies bedeutet im Klartext, dass sich die Träger der Caritas die international anerkannten Standards guter und verantwortungsvoller Unternehmensführung im Rahmen einer Selbstverpflichtung zu eigen machen sollen. Damit soll das Ver-

\begin{tabular}{|l|c|c|}
\hline & 2006 & 2009 \\
\hline $\begin{array}{l}\text { Ehrenamt in der operativen Geschäftsführung ist } \\
\text { vorhanden }\end{array}$ & 24 & 18 \\
\hline Aufsichtsorgan ist vorhanden & 63 & 69 \\
\hline $\begin{array}{l}\text { Mindestens drei Sitzungen des Aufsichtsgremiums } \\
\text { pro Jahr }\end{array}$ & 77 & 83 \\
\hline Maximal zehn Mitglieder im Aufsichtsgremium & 80 & 82 \\
\hline $\begin{array}{l}\text { Zahlung einer Aufwandsentschädigung an die Mit- } \\
\text { glieder des Aufsichtsgremiums }\end{array}$ & 23 & 30 \\
\hline $\begin{array}{l}\text { Abschluss Vermögensschadenshaftpflichtversicherung } \\
\text { für die Mitglieder des Aufsichtsgremiums }\end{array}$ & 78 & 86 \\
\hline
\end{tabular}

Tabelle: Struktur und Praxis der Aufsicht bei den Rechtsträgern der Caritas Quelle: Trägerstrukturerhebung des Deutschen Caritasverbandes 2006 (Stichtag 1.12.2006), 2009 (Stichtag 30.1.2009).

langfristig zu sichern. Die ethische Grundorientierung des kirchlichen Handelns impliziert auch einen verantwortungsvollen Umgang mit den eigenen Ressourcen. Angesprochen werden hier eine Vielzahl von Instrumenten: von der Einrichtung eines funktionsfähigen Rechnungswesens bis hin zur Marktanalyse. Besonders hervorzuheben ist die Imple-

\section{»Die Information und Qualifizierung von Aufsichtsgremien muss in den Mittelpunkt der verbandlichen Arbeit rücken «}

trauen der Eigentümer (Gesellschafter, Mitglieder, Stifter), der Klienten, Patienten, Angehörigen, der Mitarbeitenden und der Öffentlichkeit in die Leitung und Überwachung von Caritas-Trägern gestärkt werden. Hauptzielsetzung ist dabei, durch ein höheres Maß an Transparenz die Kontroll- und Überwachungsfunktion im Unternehmen zu stärken.

\section{Empfehlungen}

Die erwähnte Arbeitshilfe fordert zum einen Professionalität in der Geschäftsführung. Die Beachtung betriebswirtschaftlicher Grundsätze ist dabei kein Selbstzweck, sondern sie ist erforderlich, um den kirchlichen Sendungsauftrag der Einrichtungen und Dienste der Caritas mentierung eines Risikomanagementsystems bei den Trägern. Es ist Pflicht jeder Geschäftsführung Risiken zu erkennen und entsprechende Gegenmaßnahmen rechtzeitig zu ergreifen. (2)

Jeder katholische Rechtsträger sollte zweitens verpflichtet sein in seiner Satzung oder im Gesellschaftsvertrag neben dem Vorstand oder der Geschäftsführung ein Aufsichtsgremium zu installieren. Die Einrichtung eines eigenständigen Aufsichtsgremiums, das in der Lage ist, eine effektive wirtschaftliche Aufsicht und Kontrolle auszuüben, stellt eine ganz wesentliche strategische Weichenstellung für einen Rechtsträger dar. Die Mitgliederversammlung eines Vereins erfüllt in der Regel nicht die Voraussetzung für eine qualifizierte Aufsichtstätigkeit, es sei denn, der Verein besteht nur aus wenigen Mitgliedern, die sich in ihrer Gesamtheit regelmäßig, mehr als einmal jährlich treffen und aufgrund ihrer Zusammensetzung qualifizierte Aufsichtstätigkeiten wahrnehmen können. Gleiches gilt für einen ehrenamtlich geprägten Vorstand. Hier liegt in vielen Vereinen eine Gemengelage vor, die einer effektiven wirtschaftlichen Aufsicht entgegensteht. Die ehrenamtlichen Vorstandsmitglieder machen oftmals beides Geschäftsführung und Aufsicht - in unterschiedlichen Anteilen und fühlen sich aufgrund der hohen auch zeitlichen Anforderungen im Bereich der Geschäftsführung nicht unbedingt wohl. Mittels einer klaren Trennung zwischen Geschäftsführung und Aufsicht werden beide Aufgaben wirkungsvoller wahrgenommen und es herrscht Klarheit bezüglich der Haftung.

Das Aufsichtsgremium hat bestimmte Qualitätsanforderungen zu erfüllen. Das Aufsichtsgremium soll aus mindestens zwei (»Vieraugenprinzip«), besser drei oder noch mehr Personen bestehen. Die maximale Größe dieses Gremiums soll der fachlichen und wirtschaftlichen Bedeutung des Trägers entsprechen.

Ein wesentliches Merkmal ist die Unabhängigkeit des Aufsichtsgremiums. Bei der Zusammensetzung ist deshalb darauf $\mathrm{zu}$ achten, dass Interessenkonflikte möglichst ausgeschlossen sind. Ausschlusskriterien für die Mitarbeit in einem Aufsichtsgremium wären demnach:

- Mitglieder des Vorstandes oder der Geschäftsführung

- verwandtschaftliche Beziehungen zu Mitgliedern des Vorstandes oder der Geschäftsführung und zu Mitarbeiterinnen oder Mitarbeitern, die der unmittelbaren Kontrolle und Aufsicht unterliegen 
- Personen, die beim Wirtschaftsprüfer und oder Steuerberater beschäftigt sind

- Personen, die persönlich oder aufgrund ihrer Funktion in einer Wettbewerbsbeziehung zu dem Träger stehen

Betroffene, beispielsweise Bewohner eines Heimes oder deren Angehörige, und Personen, die persönlich oder aufgrund ihrer Funktion in einer geschäftlichen Beziehung zum Träger stehen (z. B. Vertreter der Hausbank) sollten nur bei NichtVorliegen von Interessenkollisionen aufgenommen werden und nicht die Mehrheit im Gremium bilden. Für die Lobby- und Beziehungsarbeit sollten andere Formen der Zusammenarbeit und Mitarbeit (z. B. die Einrichtung eines Kuratoriums) gewählt werden.

Die Mitglieder des Aufsichtsgremiums sollen die notwendigen Fach- und Sachkenntnisse mitbringen, die aufgrund der Aufgabenstellung des Trägers erforderlich sind. Bei der Zusammensetzung sollte deshalb auf unterschiedliche Kompetenzen Wert gelegt werden. Die ökonomische Kompetenz sollte ebenso berücksichtigt werden wie Kenntnisse über das operative Geschäft des Trä- gers. Die Anforderungsprofile können in der Satzung oder im Gesellschaftsvertrag festgelegt werden. Vermieden werden sollten auf jeden Fall die Wahl oder die Berufung "verdienter « Persönlichkeiten oder sogenannte Gefälligkeitsberufungen.

\section{Stand der Umsetzung}

Seit 2006 zeigt sich ein merklicher Fortschritt (siehe Tabelle): Der Anteil der Rechtsträger, die nach eigener Auskunft über ein eigenständiges in Satzung oder Gesellschaftsvertrag verankertes Aufsichtsgremium verfügen, ist von 63 Prozent auf 69 Prozent (Anfang 2009) angestiegen. (3)

Gleichzeitig ist der Anteil der Rechtsträger, bei denen Ehrenamtliche in der operativen Geschäftsführung vertreten sind, merklich zurückgegangen. Aus der Größe und Sitzungsfrequenz des Gremiums lassen sich zumindest Anhaltspunkte für die Effektivität der Aufsicht ableiten. Weniger als drei Sitzungen pro Jahr reichen für eine wirksame Aufsichtstätigkeit sicherlich nicht aus. Bei lediglich 17 Prozent der Rechtsträger ist dies der Fall, 2006 lag der Wert bei 23 Prozent. Mammutgremien mit mehr als zehn Mitgliedern sind ebenfalls nicht förderlich für die Qualität der Aufsicht, da das einzelne Mitglied in solch einem Setting nicht ausreichend gefordert wird. Die deutliche Mehrheit (82 Prozent) der Aufsichtsgremien arbeitet mit einer Mitgliederzahl, die zehn nicht überschreitet.

Die Gewinnung kompetenter Mitglieder für die Aufsichtsgremien ist der entscheidende Faktor für die Qualität der Aufsicht. Anreize in Form einer ausreichend ausgestatteten Aufwandsentschädigung und einer Absicherung der ehrenamtlichen Tätigkeit können hier hilfreich sein. Nach wie vor ist der Anteil der Rechtsträger, die eine Aufwandsentschädigung zahlen, mit 30 Prozent recht gering. Der Abschluss einer Vermögensschadenshaftpflichtversicherung bietet Sicherheit für das einzelne Organmitglied, aber auch für den Träger selbst im Falle eines Schadensfalles. Es gilt der Grundsatz »Ehrenamt schützt nicht vor Haftung. « Vor diesem Hintergrund ist es

\section{Was der Dachverband für seine Mitgliedsorganisationen tun kann}

Die Rechtsträger und deren Organvertreter benötigen für die Weiterentwicklung der Organstrukturen und deren Umsetzung in die Praxis verbandliche Unterstützung. Dies ist ein Feld, was bisher noch nicht ausreichend als verbandliches Aktionsfeld entdeckt worden ist. Die folgenden Umsetzungsschneisen gibt es:

Information: Die Wirksamkeit der Aufsichtstätigkeit hängt entscheidend von der Menge und Qualität der Informationen ab, die Mitgliedern von Aufsichtsgremien an die Hand gegeben werden. Für die Beurteilung von strategischen Konzepten der Geschäftsführung und fachlichen Fragen benötigen die Mitglieder des Aufsichtsgremiums neben Informationen aus dem internen Berichtswesens des Unternehmens auch ein ausreichendes Maß an Informationen aus der verbandlichen und sozialwirtschaftlichen Praxis. Die Mitglieder von Aufsichtsgremien der Caritas müssen deshalb noch weit stärker als Zielgruppe der verbandlichen Arbeit entdeckt werden. Bestehende Verbandsmedien wie die Zeitschrift »neue caritas « werden in Zukunft stärker dafür genutzt.

Qualifizierung: Die ausreichende Qualifikation der Mitglieder von Aufsichtsgremien ist essentiell für die Implementierung wirksamer Aufsichtsstrukturen. Seit dem Jahr 2004 werden regelmäßig Fortbildungen zu den Rechten und Pflichten von Aufsichtsgremien durch die Fortbildungsakademie des Deutschen Caritasverbandes angeboten. Dieses Angebot wird mo- dular erweitert und gemeinsam mit den Diözesan-Caritasverbänden umgesetzt. Die Informations- und Qualifizierungskampagne für die Mitglieder von Aufsichtsgremien der Caritas macht jedoch nur dann Sinn, wenn sie Teil einer verbandspolitischen Strategie sind, welche die Implementierung effektiver Aufsichts- und Kontrollstrukturen als ein wesentliches Qualitätsmerkmal der Caritas ansieht und aus dieser Einsicht heraus auf eine bessere Einbindung und Befähigung der Mitglieder von Aufsichtsgremien setzt.

Umsetzung in verbandliches und Satzungsrecht: Der Umsetzungsgrad der Arbeitshilfe 182 »Soziale Einrichtungen in katholischer Trägerschaft und wirtschaftliche Aufsicht « und dessen Nachhaltigkeit kann deutlich erhöht werden, wenn zentrale Aussagen der Arbeitshilfe in verbandliches und Satzungsrecht umgesetzt werden. Dafür bestehen mehrere Möglichkeiten. Sie reichen von der Verabschiedung eines spezifischen Corporate Governance Kodex für die eigene Diözese (siehe hierzu das Beispiel des Caritasverbandes der Diözese Rottenburg-Stuttgart) bis zur Vorgabe einer Mustersatzung für Caritas-Träger in einer Diözese, die innerhalb eines bestimmten Zeitraumes seitens der Träger umgesetzt werden muss. Bei der Umsetzung in verbandliches Recht sind die Kompetenz der Diözesan-Caritasverbände und ein intensiver Erfahrungsaustausch unter ihnen gefragt.

Christopher Bangert 
erfreulich, dass der Anteil der Aufsichtsgremien mit vorhandener Vermögensschadenshaftpflichtversicherung im Vergleich zu 2006 nochmals deutlich gestiegen ist.

\section{Ausblick}

Trotz der positiven Tendenzen hinsichtlich des Umsetzungsgrades bleibt für die Caritas noch genug zu tun: Zur Etablierung effektiver Aufsichtsstrukturen gehört nicht allein die Einrichtung eines Aufsichtsorgans, sondern Aufsicht muss auch gelebt werden.

Damit rücken die Themen Information und Qualifizierung von Aufsichtsgremien in den Mittelpunkt der verbandlichen Arbeit. Aufsichtsorgane sollen zu strategischen Mitdenkern werden, die auf Augenhöhe mit der Geschäftsführung argu- mentieren und gleichzeitig ihrer Überwachungsfunktion gerecht werden. Auch bei börsennotierten Unternehmen ist dies eine Baustelle.

Die Gesellschaft macht derweil nicht halt. An die Caritas werden zunehmend Transparenzanforderungen herangetragen. Zu nennen sind hier der Entwurf der neuen Leitlinien des Deutschen Zentralinstituts für soziale Fragen, demgemäß für den Erwerb des Siegels ein weit höheres Maß an interner Aufsicht und an Transparenz nach außen vorgehalten werden muss.

Die gemeinnützigen Sozialunternehmen müssen sich darauf einstellen, dass sie in Zukunft mehr Rechenschaft in wirtschaftlichen und Leistungsbelangen gegenüber der Öffentlichkeit ablegen müssen. Die Transparenzinitiative von Transparency Deutschland ist dafür ein aktuelles Beispiel.

\section{Anmerkungen}

(1) Vgl. Arbeitshilfe 182 des Verbandes der Diözesen Deutschlands und der Kommission für caritative Fragen der Deutschen Bischofskonferenz ( $\mathrm{Hg}$.): Soziale Einrichtungen in katholischer Trägerschaft und wirtschaftliche Aufsicht, März 2007, Seite 16 f. (zu finden im Internet unter www.dbk.de Arbeitshilfen).

(2) Vgl. Leitlinien für Unternehmerisches Handeln der Caritas, Zeitschrift »neue caritas «, 20/2008, S. $31 \mathrm{ff}$.

(3) Ein ausführlicher Beitrag mit dem Titel »Trägerstrukturen im Wandel « findet sich im neuen "caritas Jahrbuch $2010 \ll$, S. $159 \mathrm{ff}$.

\section{Einführung in die Organisationslehre und Organisationsstrukturen im Sozialwesen}



Bitte bestellen Sie im Buchhandel oder versandkostenfrei unter $\downarrow$ www.nomos-shop.de

\author{
Organisationen und Organisationsstrukturen \\ im Sozialwesen \\ Von Dipl.-Soz.-Wiss. Patrick Schupp \\ 2010, 229 S., brosch., 24,90 €, ISBN 978-3-8329-5843-5
}

Dieses Einführungswerk verschafft einen Einblick in die Rahmenbedingungen der Tätigkeit von Organisationen im Sozialwesen sowie in die Grundthemen der Organisationslehre und der Arbeit in Organisationen. Es eignet sich als Basisliteratur im Studium der Sozialen Arbeit oder Sozialwissenschaften, aber auch für andere Berufe mit Bezug zum Sozialwesen.

Ziel ist es, klassische und neuere Themen der Organisationslehre und -soziologie, Ergebnisse aus der Nonprofit- und Verbände-Forschung sowie verwaltungs- und politikwissenschaftliche Erkenntnisse zusammenzutragen, welche für die Tätigkeit in Organisationen und für die Bewertung und Gestaltung organisatorischer Veränderungen im Sozialwesen von zentraler Bedeutung sind.

Zu den Schwerpunktthemen zählen u.a. die Rahmenbedingungen und Binnenstruktur von Einrichtungen und Trägern im Sozialwesen, die Bedeutung der Mitarbeiter (Motivation und Führung), aktuelle Veränderungen und Modernisierungstendenzen im Sozialwesen. 